\title{
Soluble Endoglin (sCD105) as a Novel Biomarker for Detecting Aggressive Prostate Cancer
}

\author{
ADRIANA C. VIDAL ${ }^{1}$, FRANK DUONG ${ }^{2}$, LAUREN E. HOWARD ${ }^{3,4}$, EMILY WIGGINS ${ }^{4}$, \\ STEPHEN J. FREEDLAND ${ }^{1,4}$, NEIL A. BHOWMICK ${ }^{2}$ and JUN GONG ${ }^{5}$ \\ ${ }^{1}$ Division of Urology, Department of Surgery, Samuel Oschin Comprehensive Cancer Institute, \\ Cedars-Sinai Medical Center, Los Angeles, CA, U.S.A.; \\ ${ }^{2}$ Department of Medicine, Samuel Oschin Comprehensive Cancer Institute, \\ Cedars-Sinai Medical Center, Los Angeles, CA, U.S.A.; \\ ${ }^{3}$ Duke Cancer Institute, Duke University School of Medicine, Durham, NC, U.S.A.; \\ ${ }^{4}$ Surgery Section, Durham VA Medical Center, Durham, NC, U.S.A.; \\ ${ }^{5}$ Division of Hematology/Oncology, Department of Medicine, Samuel Oschin Comprehensive Cancer Institute, \\ Cedars-Sinai Medical Center, Los Angeles, CA, U.S.A.
}

\begin{abstract}
Background/Aim: We have previously found elevated levels of endoglin (CD105) in the prostate cancer $(P C)$ tissue of men with poor prognosis, compared to men with indolent disease. Herein, we examined whether plasma levels of the soluble form of CD105 (sCD105) differ according to the $P C$ grade at diagnosis. Patients and Methods: We measured sCD105 in 73 subjects with biopsy-confirmed PC at the Durham, North Carolina, Veteran Affairs Health System. The association between sCD105 and intermediate/high-grade PC risk [Gleason Group (GG) 2-5 vs. 1] was examined using regression models. Results: Of 73 men, 27 had low-grade PC and 46 high-grade PC. Higher GG was linked to lower sCD105 (GG1: $6938 \mathrm{pg} / \mathrm{ml}, \mathrm{GG2}-3$ : $6150 \mathrm{pg} / \mathrm{ml}, \mathrm{GG4}-5: 5554$ $\mathrm{pg} / \mathrm{ml} ; \mathrm{p}=0.012)$. On multivariable analysis, lower sCD105 was associated with increased high-grade $P C$ risk $\left(O R_{\text {per }} 1000\right.$ units $=1.33, p=0.028)$. Conclusion: Lower $s C D 105$ levels were associated with intermediate and high-risk PC. Further investigation is warranted in a larger PC cohort.
\end{abstract}

Prostate cancer (PC) is the second most common cancer in USA men and the second leading cause of cancer death among USA men with an estimated 174,650 new cases and

This article is freely accessible online.

Correspondence to: Adriana C. Vidal, Ph.D., Division of Urology, Department of Surgery, Samuel Oschin Comprehensive Cancer Institute, Cedars-Sinai Medical Center, Los Angeles, CA 90048, U.S.A. Tel: +1 3104234972, e-mail: adriana.vidal@cshs.org

Key Words: Prostate cancer, prostate biopsy, endoglin, high-grade prostate cancer, $\mathrm{sCD} 105$. approximately 31,620 deaths in 2019 (1). The natural history of PC can vary and ranges from indolent, low-risk disease where patients are more likely to die from competing comorbidities, to metastatic-lethal disease with a median time to death of only a few years (2). Although prostatespecific antigen (PSA) remains the most established and widely used blood-based biomarker for detecting PC and monitoring therapeutic response, it is far from ideal with post false positives and false negatives. Thus, identification of novel biomarkers, particularly those that could stratify indolent from aggressive disease would be useful for prognostication and guiding management strategies in PC.

A prior study examining cytokines in the prostatic fluid of PC patients who underwent radical prostatectomy has found increased expression of angiogenesis cytokines (3). Among the top 20 upregulated molecules in the prostatic fluid was endoglin (CD105), a type I homodimeric integral transmembrane glycoprotein and accessory TGF- $\beta$ receptor (3). El Gohary et al. (4) have found that endoglin was expressed in the PC microvasculature of radical prostatectomy specimens and that high endoglin microvessel count correlated with shorter survival. It was later found that higher preoperative plasma levels of CD105 were associated with poor clinical outcomes of PC such as higher PSA values, higher Gleason scores, and biochemical recurrence after radical prostatectomy (5). The expression of CD105 on cancer associated fibroblasts has been demonstrated to be elevated with and contribute to $\mathrm{PC}$ resistance to androgen targeted therapy (6). A study has measured endoglin in urine and serum samples of men at high risk of PC (7). In that study, urinary levels of endoglin were positively associated with PC risk, and although there were no differences in serum endoglin between controls and PC cases, higher serum levels of endoglin were associated with higher pathological stage (7). 
Our group has recently demonstrated that circulating plasma levels of the cleaved extracellular domain of endoglin (sCD105) was inversely correlated with CD105 expression in the stromal compartment of PC tissues (8). Thus, lower blood sCD105 concentrations represented increased stromal expression of CD105 and were a predictor of PC progression after radical prostatectomy (8). Based on these findings and on the observation that this has not been tested yet in men undergoing biopsy, we examined plasma sCD105 in a separate cohort of patients who were scheduled for a PC biopsy to determine the potential of sCD105 as a marker for more aggressive disease at prostate biopsy. We hypothesized that lower circulating levels of sCD105 would be associated with high-grade PC.

\section{Patients and Methods}

Study population. After obtaining Institutional Review Board approval, men undergoing prostate needle biopsy for elevated PSA and/or abnormal digital rectal examination (DRE) between 2007 and 2014 at the Durham Veterans Affairs (VA) Health Care System were recruited to participate in an ongoing study. Men were at least 18 years of age and had a PSA test within 12 months prior to enrollment. All men signed a written informed consent form. We randomly selected 100 patients with a positive prostate biopsy belonging to each of 3 the PC risk groups: 1. D'Amico low-risk, 2. D'Amico high-risk, localized, and 3. D'Amico high-risk, metastatic patients. Patients were evenly distributed in each group and matched by race, though substitutions were made if there were not enough patients in each group in order to get to 100 . Blood was collected prior to biopsy and sCD105 was measured in plasma diluted 1:100 in assay buffer provided in the CD105 (soluble) human instant ELISA kit (BMS2105INST, eBioscience, Waltham, MA, USA). sCD105 was measured per the manufacturer's directions in $\mathrm{pg} / \mathrm{ml}$. Of the 100 blood samples analyzed, 73 had sCD105 levels within the assay range, i.e. samples did yield results within the standard curve. These 73 patients, of whom 16 had metastatic PC, had not undergone any therapy at the time of biopsy results.

Statistical analyses. Grade was assigned using the five-grade group system where low-grade disease was defined as grade group (GG) 1 and high-grade PC as GG 2-5 (9). sCD105 was compared across GGs (1, 2-3, 4-5) using a Kruskal-Wallis test and across D'Amico risk groups (10) [low (i.e. indolent - watchful waiting) vs. intermediate or high (i.e. potentially aggressive - treatment usually indicated)] using a Wilcoxon rank-sum test. The association between sCD105 and odds of high-grade PC (GG 2-5 vs. 1) were tested using logistic regression, adjusted for age, race, PSA, prostate volume, year of biopsy, and digital rectal exam findings. All analyses were performed using SAS 9.3 (SAS Institute, Inc., Cary, NC, USA).

\section{Results}

Table I shows the characteristics of the study participants. There were 27 men with low-grade PC and 46 men with high-grade PC. Men with high-grade PC had higher pre- biopsy PSA values (median PSA: $18.7 \mathrm{ng} / \mathrm{ml}$ ) compared to men with low-grade PC (median PSA: $6.3 \mathrm{ng} / \mathrm{ml}$ ). There were 44 (96\%) men with high-grade PC in the D'Amico high risk group compared to $7(26 \%)$ men with low-grade PC. Median sCD105 levels were lower in men with high-grade PC $(5804 \mathrm{pg} / \mathrm{ml})$ compared to those of men with low-grade PC (6939 pg/ml) (Table I).

Men with higher GG had lower values of sCD105 (GG 1: median 6938 pg/ml, GG 2-3: 6150 pg/ml, GG 4-5: 5554 $\mathrm{pg} / \mathrm{ml} ; p=0.012)$. Similarly, men with D'Amico intermediate or high-risk disease had lower levels of sCD105 than those with low risk (median 5958 vs. 8506 pg/ml, $p=0.006$ ) (Table II).

On univariable analysis, lower sCD105 was associated with increased odds of high-grade PC (HR per 1000 units $=1.28,95 \% \mathrm{CI}=1.05-1.54, p=0.003)$. After adjusting for age, race, PSA, TRUS prostate volume, digital rectal exam findings, and year of biopsy, the association was similar (OR per 1000 units $=1.33,95 \% \mathrm{CI}=1.03-1.72, p=0.028$ ) (Table III).

\section{Discussion}

In this study, we confirm that lower levels of the soluble form of endoglin, sCD105, were associated with intermediate and high-grade PC at biopsy, as we hypothesized. The present results indicate for the first time that lower circulating levels of sCD105 are associated with more aggressive PC at biopsy, and agree with previous data from our laboratory which showed that lower sCD105 plasma levels prior to radical prostatectomy were associated with recurrent disease within 5 years (8). If validated, sCD105 may be a novel blood-based biomarker to detect aggressive PC.

Our results are in conflict with previous data among 69 men diagnosed with PC at the Brady Urology Clinic at John Hopkins (7). In that study, increased endoglin serum levels were associated with non-organ confined PC compared to organ-confined PC ( $p=0.004)$ (7). Of note, in that study there were no differences in serum endoglin between controls and PC cases, and it was not specified whether the cleaved form of endoglin was measured (7). A larger study among 425 men undergoing radical prostatectomy at the University of Texas Southwestern Medical Center at Dallas (5), used the same commercial ELISA kit (R\&D Systems) to measure CD105 as in the Fujita study (7), and also found that higher pre-operative plasma levels of CD105 were associated with worse PC outcomes (5). We used a different ELISA kit (eBioscience) to quantify endoglin in plasma. Disparate results may be due to the specific ELISA tests' anti-CD105 antibodies used by the commercial kits. Another alternative explanation is that endoglin levels may be affected by archival storage time. Furthermore, patient characteristics were different across studies and the subjects in our study had a higher median PSA compared to those in Fujita et al. 
Table I. Baseline patient characteristics.

\begin{tabular}{|c|c|c|c|c|}
\hline & $\begin{array}{l}\text { High-grade } \\
(\mathrm{GG} 2-5) \\
(\mathrm{N}=46)\end{array}$ & $\begin{array}{l}\text { Low-grade } \\
\quad(\mathrm{GG} 1) \\
(\mathrm{N}=27)\end{array}$ & $\begin{array}{c}\text { Total } \\
(\mathrm{N}=73)\end{array}$ & $p$-Value \\
\hline Age & & & & $0.284^{1}$ \\
\hline Median & 64 & 63 & 63 & \\
\hline Q1, Q3 & 60,68 & 59,66 & 59,66 & \\
\hline Year & & & & $0.004^{1}$ \\
\hline Median & 2010 & 2012 & 2011 & \\
\hline Q1, Q3 & 2009,2011 & 2010,2013 & 2009,2012 & \\
\hline Race & & & & $0.302^{2}$ \\
\hline Black & $25(54 \%)$ & $18(67 \%)$ & $43(59 \%)$ & \\
\hline White & $21(46 \%)$ & $9(33 \%)$ & $30(41 \%)$ & \\
\hline $\mathrm{PSA}, \mathrm{ng} / \mathrm{ml}$ & & & & $0.001^{1}$ \\
\hline Median & 18.7 & 6.3 & 10.4 & \\
\hline Q1, Q3 & $7.5,41.0$ & $5.1,10.4$ & $5.8,27.7$ & \\
\hline Prostate volume, $\mathrm{ml}$ & & & & $0.139^{1}$ \\
\hline Median & 34.0 & 40.6 & 35.0 & \\
\hline Q1, Q3 & $24.6,47.5$ & $28.4,60.0$ & $27.0,51.0$ & \\
\hline Digital rectal exam & & & & $0.004^{2}$ \\
\hline Normal & $20(43 \%)$ & $21(78 \%)$ & $41(56 \%)$ & \\
\hline Abnormal/suspicious & $26(57 \%)$ & $6(22 \%)$ & $32(44 \%)$ & \\
\hline D'Amico risk group & & & & $<0.001^{3}$ \\
\hline Low risk & $0(0 \%)$ & $18(67 \%)$ & $18(25 \%)$ & \\
\hline Intermediate risk & $2(4 \%)$ & $2(7 \%)$ & $4(5 \%)$ & \\
\hline High risk & $44(96 \%)$ & $7(26 \%)$ & $51(70 \%)$ & \\
\hline sCD105 & & & & $0.004^{1}$ \\
\hline $\operatorname{Median}(\mathrm{pg} / \mathrm{ml})$ & 5804 & 6939 & 6362 & \\
\hline Q1, Q3 & 3971,7285 & 5958,9208 & 4458,8439 & \\
\hline
\end{tabular}

${ }^{1}$ Wilcoxon; ${ }^{2}$ Chi-Square; ${ }^{3}$ Fisher Exact.

(7) and a higher proportion of low-grade PCs compared to both Fujita et al. (7) and Svatek et al. (5).

A Swedish study that analyzed tissue microarrays (TMAs) of men diagnosed with PC found that increased endoglin vascular density in the TMAs was associated with shorter cancer-specific survival (11). This relationship between higher tissue endoglin vascular density and shorter PC survival levels was also supported by a separate group (4). Our group has recently shown that circulating plasma levels of the cleaved extracellular domain of endoglin (sCD105) inversely correlated with endoglin (CD105) expressed in the stromal compartment of PC tissues (8). Therefore, our findings, in this study, are consistent with these results in supporting that lower plasma sCD105 concentrations (i.e. high tissue CD105 levels) potentially correlate with more aggressive PC.

Endoglin has been proposed as a potential biomarker across several malignancies (12-14), including PC (5). Not only did we demonstrate that plasma sCD105 is associated with the pathological GG, but also that circulating levels of sCD105 were associated with PC risk group. The D'Amico risk group is a historical classification that is standardly used in stratifying low, intermediate, and high-risk patients with
Table II. Analysis of CD105 in Biopsy data. Median, p25, and p75 of sCD105 by grade group and D'Amico risk group.

\begin{tabular}{lcc}
\hline & $\mathrm{n}$ & $\begin{array}{c}\text { sCD105 }(\mathrm{pg} / \mathrm{ml}) \\
\text { Median }(\mathrm{Q} 1, \mathrm{Q} 3)\end{array}$ \\
\hline Gleason score & & \\
1 & 27 & $6938(5958,9208)$ \\
$2-3$ & 19 & $6150(4458,7294)$ \\
$4-5$ & 27 & $5554(3438,7285)$ \\
$p$-Value* & & 0.012 \\
D'Amico risk group & 18 & $8506(6688,9362)$ \\
$\quad$ Low & 55 & $5958(4147,7294)$ \\
Intermediate/High & & 0.006 \\
$p$-Value** & & \\
\hline
\end{tabular}

*By Kruskal-Wallis test; **By Wilcoxon rank-sum test.

Table III. Association between sCD105 and Gleason score (GG 2-5 vs. GG 1).

\begin{tabular}{lccc}
\hline & OR & $95 \% \mathrm{CI}$ & $p$-Value \\
\hline Univariable & 1.28 & $1.05-1.54$ & 0.003 \\
Multivariable* & 1.33 & $1.03-1.72$ & 0.028 \\
\hline
\end{tabular}

Odds ratio is modeled per 1000 units of CD105. *Adjusted for age, race, PSA, TRUS prostate volume, digital rectal exam findings, and year of biopsy.

PC but is dependent on prostate tumor biopsies for calculation (10). In non-metastatic PC, such a classification scheme is emphasized in current treatment guidelines seeking to tailor management strategies according to risk [e.g., active surveillance is recommended in low risk disease while definitive therapy is often recommended in those with intermediate-high risk disease and select patient factors, as in 2019 NCCN Guidelines for Prostate Cancer (15)]. As such, we are presenting a provocative blood-based biomarker that is associated with histologic grade and a traditional risk score that may complement the results of a PSA test. Further validation in larger, prospective cohorts of PC patients is warranted to establish whether plasma SCD105 can serve as a novel blood-based biomarker beyond PSA that could enhance our ability to prognosticate and select appropriate management strategies in PC.

Endoglin may also represent a viable target to sensitize PC to various therapeutic modalities. Our group has previously demonstrated that radiation-resistance was mediated, in part, by CD105 downstream of the bone morphogenetic protein and transforming growth factor- $\beta$ signaling whereas antagonizing CD105 with a new anti-angiogenic monoclonal antibody targeting CD105, TRC105, sensitized prostate 
tumors to the antitumor effects of radiation therapy in preclinical PC models in a p53-dependent manner (16).

This study has several strengths and limitations. Although men were already scheduled for a biopsy, blood was collected prior to a needle biopsy to measure sCD105 in plasma. More than $50 \%$ of men were black, indicating that sCD105 is a predictor of high-grade PC particularly in an often-regarded at-risk patient subgroup. Limitations include the small sample size of our cohort, which did not include controls, thus endoglin was not tested in PC-free men. Furthermore, men were at high-risk for PC and attending only one VA hospital, the Durham VA. Finally, we do not have outcome data on these men. Thus, we relied on definitions of aggressive cancer based on findings at the time of diagnosis. Further study is needed to determine whether sCD105 levels correlate with long-term clinical outcome. In conclusion, if our results are validated, sCD105 may be a novel blood-based biomarker to identify aggressive PC.

\section{Conflicts of Interest}

All Authors declared no conflicts of interest regarding this study.

\section{Authors' Contributions}

ACV, SJF and NAB designed the study. EW coordinated the selection and shipment of the patients' samples. FD conducted the experiments. LEH conducted the statistical analysis. ACV and JG drafted the manuscript. NAB and SJF revised the manuscript. All authors approved the final version of the manuscript.

\section{Acknowledgements}

NIH K24 CA160653. Adriana Vidal was supported by a Research Scholar Grant, RSG-18-018-01- CPHPS, from the American Cancer Society.

\section{References}

1 Siegel RL and Miller KD: Cancer statistics, 2019. CA Cancer J Clin 69(1): 7-34, 2019. PMID: 30620402. DOI: 10.3322/ caac. 21551

2 Sathianathen NJ, Konety BR, Crook J, Saad F and Lawrentschuk $\mathrm{N}$ : Landmarks in prostate cancer. Nat Rev Urol 15(10): 627-642, 2018. PMID: 30065357. DOI: 10.1038/s41585-018-0060-7

3 Fujita K, Ewing CM, Sokoll LJ, Elliott DJ, Cunningham M, De Marzo AM, Isaacs WB and Pavlovich CP: Cytokine profiling of prostatic fluid from cancerous prostate glands identifies cytokines associated with extent of tumor and inflammation. Prostate 68(8): 872-882, 2008. PMID: 18361406. DOI: $10.1002 /$ pros. 20755

4 El-Gohary YM, Silverman JF, Olson PR, Liu YL, Cohen JK, Miller R and Saad RS: Endoglin (cd105) and vascular endothelial growth factor as prognostic markers in prostatic adenocarcinoma. Am J Clin Pathol 127(4): 572-579, 2007. PMID: 17369132. DOI: 10.1309/X6NXYE57DLUE2NQ8
5 Svatek RS, Karam JA, Roehrborn CG, Karakiewicz PI, Slawin KM and Shariat SF: Preoperative plasma endoglin levels predict biochemical progression after radical prostatectomy. Clin Cancer Res 14(11): 3362-3366, 2008. PMID: 18519764. DOI: 10.1158/1078-0432.CCR-07-4707

6 Kato M, Placencio-Hickok VR, Madhav A and Haldar S: Heterogeneous cancer-associated fibroblast population potentiates neuroendocrine differentiation and castrate resistance in a cd105-dependent manner. Oncogene 38(5): 716-730, 2019. PMID: 30177832. DOI: 10.1038/s41388-018-0461-3

7 Fujita K, Ewing CM, Chan DY, Mangold LA, Partin AW, Isaacs WB and Pavlovich CP: Endoglin (cd105) as a urinary and serum marker of prostate cancer. Int J Cancer 124(3): 664-669, 2009. PMID: 19004009. DOI: 10.1002/ijc.24007

8 Placencio-Hickok VMA, Kim S, Duong F, Angara B, Liu Z and Bhowmick NA: Elevated scd105 in prostate cancer patient plasma correlates with non-recurrence. Endocr Relat Cancer pii: ERC-190370.R1, 2019. PMID: 31648185. DOI: 10.1530/ERC-19-0370

9 Epstein JI, Zelefsky MJ, Sjoberg DD, Nelson JB, Egevad L, Magi-Galluzzi C, Vickers AJ, Parwani AV, Reuter VE, Fine SW, Eastham JA, Wiklund P, Han M, Reddy CA, Ciezki JP, Nyberg T and Klein EA: A contemporary prostate cancer grading system: A validated alternative to the gleason score. Eur Urol 69(3): 428435, 2016. PMID: 26166626. DOI: 10.1016/j.eururo.2015.06.046

10 D'Amico AV, Whittington R, Malkowicz SB, Schultz D, Blank K, Broderick GA, Tomaszewski JE, Renshaw AA, Kaplan I, Beard CJ and Wein A: Biochemical outcome after radical prostatectomy, external beam radiation therapy, or interstitial radiation therapy for clinically localized prostate cancer. JAMA 280(11): 969-974, 1998. PMID: 9749478 . DOI: 10.1001/jama.280.11.969

11 Josefsson A, Wikstrom P, Egevad L, Granfors T, Karlberg L, Stattin $\mathrm{P}$ and Bergh A: Low endoglin vascular density and ki67 index in gleason score 6 tumours may identify prostate cancer patients suitable for surveillance. Scand J Urol Nephrol 46(4): 247-257, 2012. PMID: 22452635. DOI: 10.3109/00365599.2012.669791

12 Li C, Guo B, Wilson PB, Stewart A, Byrne G, Bundred N and Kumar S: Plasma levels of soluble cd 105 correlate with metastasis in patients with breast cancer. Int J Cancer 89(2): 122-126, 2000. PMID: 10754488. DOI: 10.1002/(sici)1097-0215(20000320)89:2< 122::aid-ijc4>3.0.co;2-m

13 Seon BK, Takahashi N, Haba A, Matsuno F, Haruta Y, She XW, Harada $\mathrm{N}$ and Tsai $\mathrm{H}$ : Angiogenesis and metastasis marker of human tumors. Rinsho Byori 49(10): 1005-1013, 2001. PMID: 11769465.

14 Tsirakis G, Pappa CA, Spanoudakis M, Chochlakis D, Alegakis A, Psarakis FE, Stratinaki M, Stathopoulos EN and Alexandrakis MG: Clinical significance of scd105 in angiogenesis and disease activity in multiple myeloma. Eur J Intern Med 23(4): 368-373, 2012. PMID: 22560388. DOI: 10.1016/j.ejim.2012.01.012

15 National Comprehensive Cancer Network. Available at: https://www.nccn.org/professionals/physician_gls/pdf/prostate.pd $\mathrm{f}$ (Last accessed on 03/02/2020)

16 Madhav A, Andres A, Duong F, Mishra R, Haldar S, Liu Z, Angara B and Gottlieb R: Antagonizing cd105 enhances radiation sensitivity in prostate cancer. Oncogene 37(32): 4385-4397, 2018. PMID: 29717261. DOI: 10.1038/s41388-018-0278-0

Received January 24, 2020

Revised January 31, 2020

Accepted February 4, 2020 